\title{
Congenital Absence of Left Atrial Appendage
}

\author{
Tawseef Dar ${ }^{1}$, Bharath Yarlagadda ${ }^{1}$, Vijendra Swarup ${ }^{2}$, Dhanunjaya Lakkireddy ${ }^{1}$ \\ ${ }^{1}$ Division of Cardiovascular Diseases, Cardiovascular Research Institute, University of Kansas Hospital E Medical Center, \\ Kansas City, KS. ${ }^{2}$ Arizona Heart Rhythm Center, Phoenix, Arizona.
}

\begin{abstract}
Isolated absence of left atrial appendage is a very rare entity and is usually encountered as an incidental finding during routine imaging for other purposes. Lately, with increasing fund of knowledge about its potential role in cardio embolic phenomena, we have seen an increased trend in the use different techniques to exclude left atrial appendage from main left atrial chamber, in an effort to alleviate the stroke risk and therefore the need for long term anticoagulation. Clinical implications of absent left atrial appendage in such patients remains a mystery.
\end{abstract}

\section{Case}

67 year old male with history of hypertension (HTN), diabetes mellitus (DM), and persistent atrial fibrillation (AF) who was being evaluated for aMAZE study underwent a Computed tomography angiography (CTA) for screening purposes. Left atrial appendage (LAA) was found to be missing [Figure 1] and patient had no previous history of surgical or percutaneous left atrial appendage exclusion.

\section{Discussion}

Left atrial appendage develops in 3rd -4th week of embryonic life from the left wall of the primary atrium and functions like a left atrium during the fetal life [Figure 2] ${ }^{[1]}$. In adults it is believed to function as a decompression chamber during elevated left atrial pressures including left ventricular systole or volume overload situations. It also contributes towards left atrial reservoir and contractile functions [2]. Its physiological and ultrastructural properties are distinct from the left atrium main chamber. Left atrial appendage (LAA) has particularly drawn attention in the last decade due to its potential role in the atrial fibrillation (AF) and thromboembolic phenomena. Ninety percent of thrombi, in the setting of non-valvular atrial fibrillation, are found in left atrial appendage ${ }^{[3]}$ and around $27 \%$ of patients presenting for repeat ablation procedures have triggers in left atrial appendage ${ }^{[4]}$. Mechanical closure/isolation of LAA for stroke prevention is, therefore, a new trend in the world of electrophysiology. Congenital absence of left atrial appendage is very rare and its impact on electromechanical properties of left atrium are not known. But, of more interest and significance would be, its clinical implications

\section{Key Words}

Decompression, Hypertension, Left Atrial Appendage. for the patient with atrial fibrillation in terms of anticoagulation or even ablation procedures. These are the areas that would need further studies and /or thorough literature review of such cases, in order to get some answers.

\section{Disclosures}

None.

\section{References}

1. Al-Saady N M, ObelO A, CammA J. Left atrial appendage: structure, function, and role in thromboembolism. Heart. 1999;82 (5):547-54

2. Tabata T, OkiT, YamadaH, IuchiA, ItoS, HoriT, KitagawaT, KatoI, KitahataH, OshitaS. Role of left atrial appendage in left atrial reservoir function as evaluated by left atrial appendage clamping during cardiac surgery. Am. J. Cardiol. 1998;81 (3):327-32

3. Blackshear J L, OdellJ A. Appendage obliteration to reduce stroke in cardiac surgical patients with atrial fibrillation. Ann. Thorac. Surg. 1996;61 (2):755-9

4. Di Biase Luigi, BurkhardtJ David, MohantyPrasant, SanchezJavier, MohantySanghamitra, HortonRodney, GallinghouseG Joseph, BaileyShane M, ZagrodzkyJason D, SantangeliPasquale, HaoSteven, HongoRichard, BeheirySalwa, ThemistoclakisSakis, BonsoAldo, RossilloAntonio, CorradoAndrea, RavieleAntonio, Al-AhmadAmin, WangPaul, CummingsJennifer E, SchweikertRobert A, PelargonioGemma, Dello RussoAntonio, CasellaMichela, SantarelliPietro, LewisWilliam R, NataleAndrea. Left atrial appendage: an underrecognized trigger site of atrial fibrillation. Circulation. 2010;122 (2):109-18

Corresponding Author

Dhanunjaya Lakkireddy,

Professor of Medicine Division of Cardiovascular Diseases, Cardiovascular Research Institute,

University of Kansas Hospital and Medical Center, 3901 Rainbow Boulevard Kansas City,

Kansas 6616 


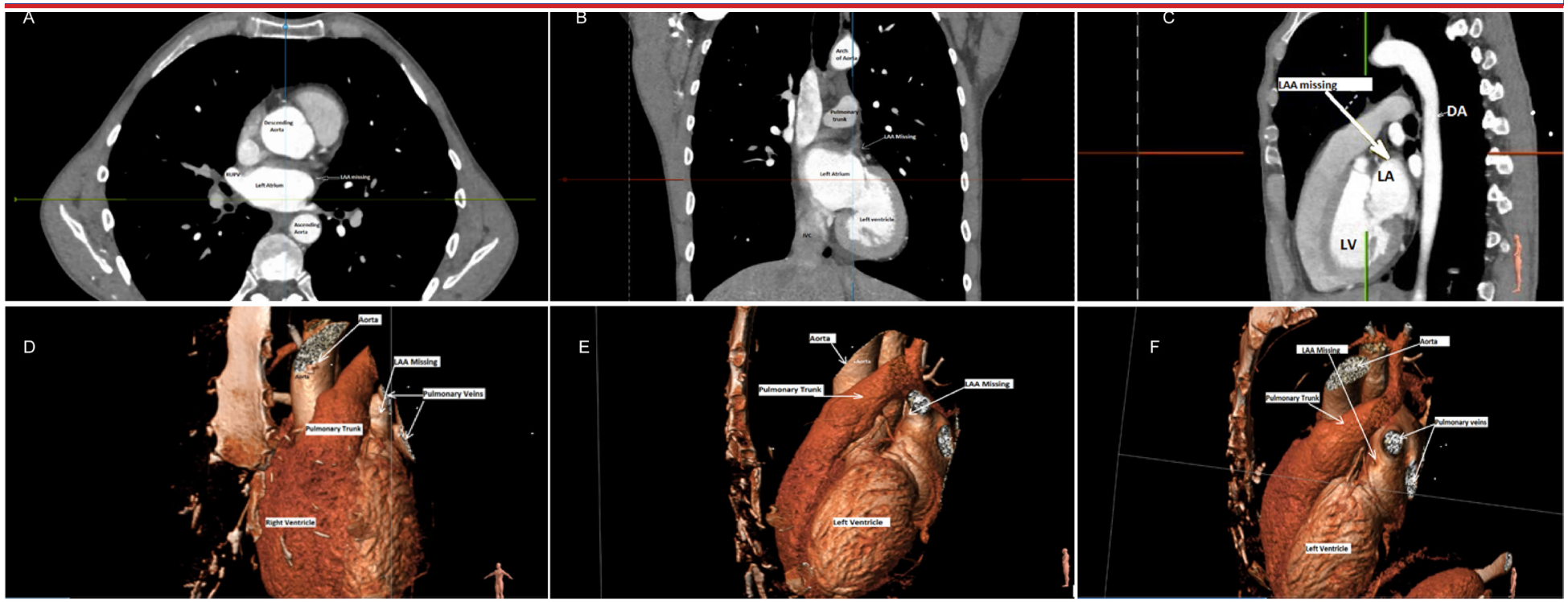

$(\mathrm{A}, \mathrm{B}, \mathrm{C}, \mathrm{D}, \mathrm{E}, \mathrm{F})$

Multidetector computed tomography imaging of the left atrium marking the structures relative to left atrial appendage using multiplanar reconstruction images (A-axial, B-coronal, C-sagittal) and 3 dimensional volume-rendered images at different angles (D,E,F). LAA: Left atrial appendage, RUPV: Right upper pulmonary vein, LV: Left Ventricle, LA: Left Atrium, DA: Descending Aorta.
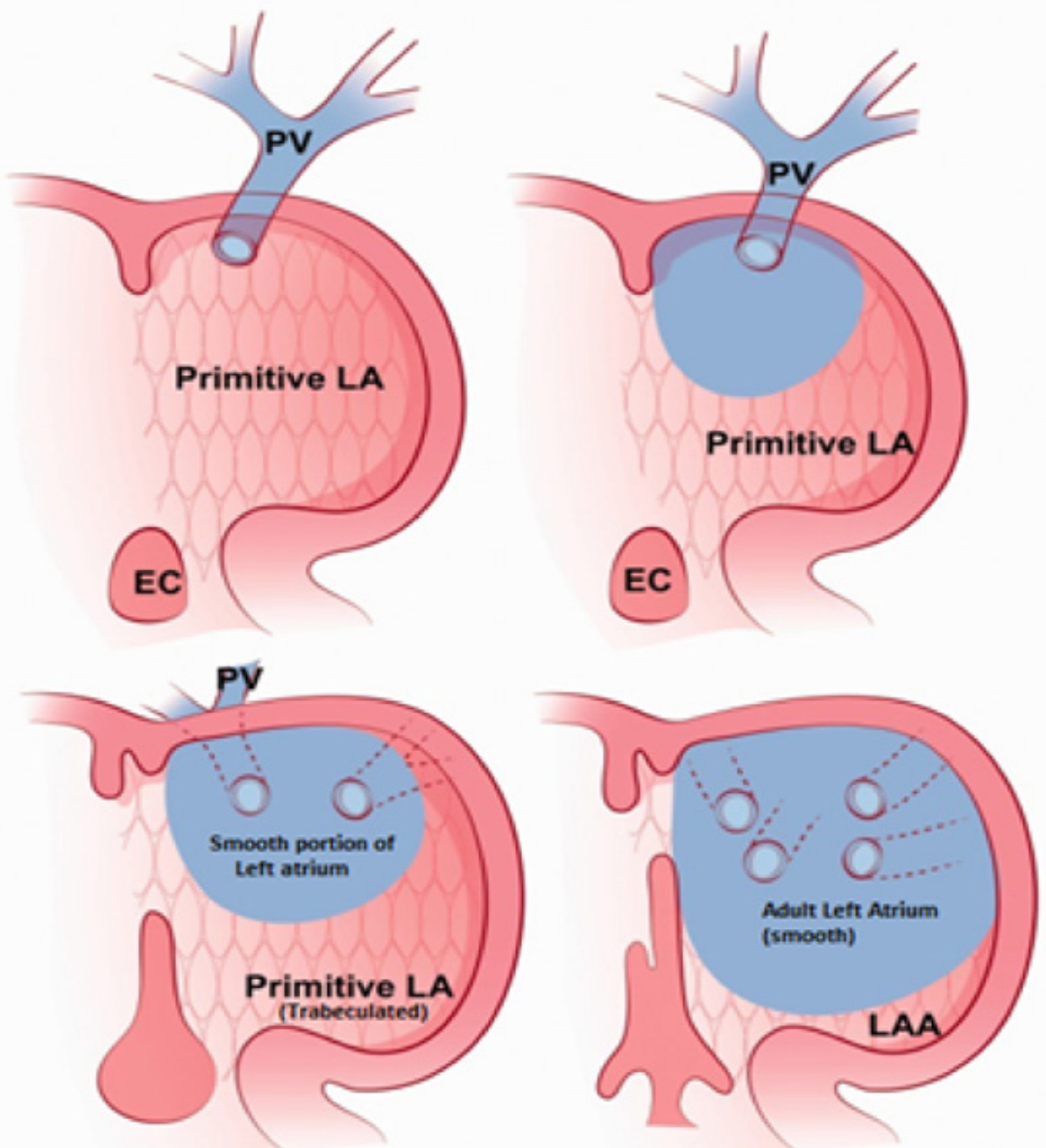

Figure 2:

Illustrating embryologic development of the left atrium and left atrial appendage (LAA). Note the primitive left atrium (trabeculated pattern) becomes left atrial appendage (LAA) and part of the pulmonary vein becomes the smooth portion of the left atrium (Blue). EC: Endocardial Cushion, LA : Left atrium, LAA: Left atrial appendage

Reproduced from Acta Radiol Short Rep. 2015 Jan; 4(1): 2047981614562443. 